\title{
É possível estabelecer uma linha de tempo de reformas curriculares no curso de Medicina da Faculdade de Medicina do ABC?
}

\author{
It is possible to set a timeline of curricular reforms in the course of Medicine \\ of Faculty of Medicine of ABC?
}

David Feder ${ }^{1}$

'Disciplina de Farmacologia, Curso de Medicina, Faculdade de Medicina do ABC (FMABC) - Santo André (SP), Brasil.

DOI: http://dx.doi.org/10.7322/abcshs.v40i3.817

\begin{abstract}
RESUMO
Introdução: A Faculdade de Medicina do ABC (FMABC) foi criada em 1969, em um período de grande expansão do ensino privado, com dois anos de ciclo básico, três anos de ciclo clínico e um de internato. Ao longo dos primeiros anos da Faculdade ocorreu um aumento progressivo do número de disciplinas e do tempo de internato para dois anos. Relato de experiência: Em 1999, iniciou-se a discussão da reforma curricular, implantada em 2002, seguindo as Diretrizes Curriculares Nacionais (DCN) de 2001, visando a integração das disciplinas e maior consistência no futuro desempenho dos graduados. Houve a criação de espaços para conteúdos multidisciplinares, favorecendo a integração das diversas disciplinas, incluindo temas humanísticos. Com a inclusão da Faculdade no Programa Nacional de Reorientação da Formação Profissional em Saúde (Pró-Saúde), em 2015, acentuaram-se as reformas curriculares, estimulando a integração do trabalho interdisciplinar e multiprofissional no sentido de aproximar academia, gestão, profissionais de saúde e comunidade. A partir de 2008, optou-se pela visão sistêmica, pelos ciclos de vida e pela introdução do Objective Structured Clinical Examination (OSCE). A partir de 2013, modificações no currículo visaram a integração do conteúdo das disciplinas do ciclo básico em módulos e o início das disciplinas do ciclo clínico a partir do segundo ano, para que o internato tivesse a duração de dois anos e meio em 2016. Com a publicação das novas DCN, em 2014, novas modificações deverão ser obrigatoriamente introduzidas no curso. Conclusão: A reforma curricular é um processo contínuo que demanda reflexão e reestruturação constante do curso. A formação dos nossos graduados tem se mostrado adequada. As nossas avaliações externas nos tranquilizam de que estamos no caminho correto.
\end{abstract}

Palavras-chave: escolas médicas; currículo; avaliação institucional.

Recebido em: 31/07/2015

Revisado em: 28/09/2015

Aprovado em: 04/10/2015

\section{ABSTRACT}

Introduction: The Faculty of Medicine of $A B C$ (FMABC), established in 1969 in a period of great expansion of private education, with two years of basic cycle, three of clinical cycle and one of internship. Over the early years of the Faculty there is a progressive increase in the number of disciplines and the internship for two years. Experience report: In 1999 began the discussion of curriculum reform, implemented in 2002, following the Brazilian Curricular Guidelines (DCN) 2001, aimed at integrating the disciplines, and greater consistency in the future performance of graduates. There was the creation of spaces for multidisciplinary content, favoring the integration of different disciplines, including humanities subjects. With the addition of the Faculty on Pró-Saúde (2015) accentuated if the curriculum reform, encouraging the integration of interdisciplinary and multidisciplinary work to bring academia, management, health professionals and community. From 2008 it was decided to systemic vision and life cycles and the introduction of the OSCE. From 2013 modifications aimed curriculum incorporating the content of the disciplines of the basic cycle in modules, beginning the disciplines of clinical cycle from the second year for the internship had a duration of 2.5 years in 2016. With the publication of new DCN in 2014 further changes should be compulsorily introduced in the course. Conclusion: Curriculum reform is an ongoing process requiring constant reflection and the ongoing restructuring. The training of our graduates have been adequate. Our external evaluations reassure us that we are on the right track.

Keywords: schools, medical; curriculum; institucional evaluation. 


\section{INTRODUÇÃO}

A Fundação do ABC (FUABC) foi instituída na forma de leis pelos municípios de Santo André, São Bernardo do Campo e São Caetano do Sul, com a finalidade de criar uma Faculdade de Medicina na Região do ABC. A solicitação para autorização de funcionamento da Faculdade de Medicina foi pleiteada pela FUABC ao Ministério da Educação (MEC) em 1968, justificada pela grande procura por vagas para cursos dessa área no Estado e pelo argumento de que a região de maior renda do país teria todas as condições para viabilizar uma escola médica. Além disso, os municípios que compunham a Região do Grande $\mathrm{ABC}$ tinham grande concentração industrial, e essa população trabalhadora seria beneficiada em termos de melhorias na assistência e higiene do trabalho, bem como atenderia ao mercado de trabalho médico, em franca expansão na região ${ }^{1}$.

Importante contextualizar o período de sua criação: a Faculdade de Medicina do ABC (FMABC) surgiu no período da Reforma Universitária de 1968, movimento que abriu condições para o surgimento de um ensino privado, uma vez que as modificações introduzidas nas universidades federais não conseguiram ampliar satisfatoriamente suas matriculas para atender à crescente demanda de acesso ${ }^{2}$. Criaram-se inúmeras instituições organizadas a partir de estabelecimentos isolados, voltados para a mera transmissão de conhecimentos de cunho marcadamente profissionalizante e distanciados da atividade de pesquisa, o que pouco contribui para a formação de um horizonte intelectual crítico para a análise da sociedade brasileira e das transformações da época ${ }^{3}$.

A estrutura curricular adotada pela FMABC, assim como pela grande maioria das faculdades brasileiras, foi influenciada pelo modelo flexneriano com ciclo básico de dois anos, ciclo clínico de três anos e um ano de internato. O ciclo clínico, como propôs Flexner, deveria se dar fundamentalmente no hospital ${ }^{4}$. A primeira turma da Faculdade iniciou suas atividades em março de 1969 em prédios emprestados pela Fundação Santo André (FSA) e, por ausência de hospital-escola, fez o internato no Hospital do Servidor Público Municipal de São Paulo com estágio nas diferentes especialidades cirúrgicas e clínicas, totalizando 17 disciplinas. A partir da segunda turma o internato em um ano passou a ter formação generalista com atividades nas quatro grandes clínicas (Clínica Médica, Cirurgia, Pediatria e Ginecologia/ Obstetrícia) realizadas no Hospital Municipal de Santo André e no Hospital Anchieta; sendo que de 1982 a 1986 havia um quinto ciclo, o de Pronto-Socorro, posteriormente incorporado na Clínica Médica.

Desde a primeira turma observamos aumento progressivo no número de especialidades incorporadas na graduação. O grande desenvolvimento ocorrido nas áreas de conhecimento concorreu para aumentar a especialização, influenciando sobremaneira a prática e o ensino médico. Este se tornou cada vez mais fragmentado, tendo como decorrência a valorização das especialidades ${ }^{5}$. A origem das disciplinas do curso de Medicina se deve à divisão do território representado pelo corpo humano em inúmeros pequenos lotes, em partes cada vez menores, do domínio científico. As disciplinas assim criadas gozavam de total autonomia para construir seu conteúdo programático, sem a integração necessária à visão holística do ser humano ${ }^{6}$.

\section{RELATO DE EXPERIÊNCIA}

A estrutura amarrada do curso não facilitava a integração básico-clínica e as disciplinas rechaçavam quaisquer propostas de mudanças na grade curricular. Acompanhando a tendência da maioria das escolas médicas brasileiras, a partir de 1984 o internato foi aumentado para um ano e meio; a partir de 1989, a duração passou a ser de dois anos. Com isso, as diferentes disciplinas do ciclo clínico tiveram sua carga horária reduzida, sendo que os alunos tinham cerca de 14 disciplinas no $3^{\circ}$ e $4^{\circ}$ anos, sem nenhuma integração.

A avaliação externa, realizada no final de 1999 por uma comissão do MEC, sugeriu diversas modificações na organização didático-pedagógica da instituição. Para discutir, organizar e implantar essas modificações, a FMABC criou uma Comissão de Reestruturação Curricular ${ }^{1}$. Em 2001, a publicação das Diretrizes Curriculares Nacionais $(\mathrm{DCN})^{7}$ foi outro grande motivador para a continuidade do processo de rediscussão do currículo, com a finalidade de adaptá-lo às novas orientações propostas ${ }^{1}$.

O Projeto de Reestruturação Curricular do Curso Médico da FMABC, concluído em 2002, sugeriu a integração das disciplinas, visando uma maior consistência e coerência no futuro desempenho dos profissionais por ela formados. Além disso, destinaram-se espaços para a criação de conteúdos multidisciplinares, favorecendo a integração das diversas disciplinas, incluindo temas humanísticos ${ }^{1}$. A primeira mudança implementada foi a redistribuição e otimização da carga horária, excluindo atividades aos sábados e incluindo um período semanal de área verde, de acordo com as possibilidades de cada série do curso. Criaram-se disciplinas eletivas que não seriam ministradas a todos os alunos, respeitando-se os interesses e as motivações individuais.

Algumas modificações ocorreram de forma incipiente (por exemplo, as propostas para o primeiro ano); outras não chegaram a ser implementadas (como a integração em bloco prevista para o segundo ano). Houve implantação da área verde, ainda que de modo heterogêneo, e verificou-se uma ampliação do número de opções de eletivas. O agrupamento 
das disciplinas por afinidade de conteúdos, proposto para o quarto ano, foi feito; no entanto, não aconteceu uma verdadeira integração entre elas ${ }^{1}$.

Para o internato, a proposta era a criação de um Ciclo de Urgências, no quinto ano, e de um Ciclo Eletivo, no sexto ano. O Ciclo de Urgências Médicas, previsto para ser inserido no quinto ano, foi incluído como subciclo da Clínica Médica. O Ciclo Eletivo no sexto ano foi implantado em 2004 e depois suprimido ${ }^{1}$.

Ou seja, mudanças essenciais não se concretizaram e a integração, compreendida na proposta de 2002 como uma etapa evolutiva para a interdisciplinaridade, não se desenvolveu como esperado.

Em 2005, a FMABC foi uma das 11 instituições privadas selecionadas para o Programa Nacional de Reorientação da Formação Profissional em Saúde (Pró-Saúde). O projeto da Faculdade destinou-se a implantar uma reformulação curricular mais efetiva. Os objetivos propostos foram: reestruturar a formação profissional do estudante de Medicina na instituição, para atender, com eficácia, às necessidades de saúde da população da região; possibilitar a reorientação das práticas médicas desde o início do curso, para as Diretrizes do Sistema Único de Saúde (SUS), em sintonia com as DCN do Curso de Graduação em Medicina; favorecer e estimular a integração do trabalho interdisciplinar e multiprofissional no sentido de aproximar academia, gestão, profissionais de saúde e comunidade. Nesta etapa de reformulação curricular institui-se em cada ano do curso algum espaço de integração: $1^{\circ}$ ano foi instituído o Módulo Fundamentos da Prática Médica; no $2^{\circ}$ ano, o Núcleo Integrador de Práticas (NIP); no $3^{\circ}$ ano foi criado o Módulo de Integração Clínico/Morfo/Funcional; no $4^{\circ}$ ano foram criadas as Práticas de Humanização do Departamento de Pediatria; para o $5^{\circ}$ e $6^{\circ}$ anos, foi criado o Ciclo de Atenção Primária à Saúde ${ }^{1}$.

Apesar das iniciativas propostas, observou-se, em 2008, que a implantação dessas modificações esbarrava em dois problemas: o currículo era eminentemente nuclear, com inclusão parcial de ciclo optativo, e encontrava-se distintamente separado por ano (do primeiro ao quarto, e estes eram separados do internato). Optou-se, a partir de então, pela visão sistêmica e pelos ciclos de vida. Algumas das modificações implantadas foram: introdução do Módulo de Metodologia de Pesquisa Científica (anteriormente uma disciplina eletiva), valorização da avaliação por conhecimentos, habilidade e atitudes. No sexto ano os alunos passaram a ser avaliados pelo Objective Structured Clinical Examination (OSCE) $)^{8}$, que é um método de avaliação prática que consiste em expor o aluno a situações que simulem procedimentos encontrados no dia a dia do médico e avaliar o seu comportamento. Também foram instituídas as atividades complementares, que são atividades curriculares de caráter independente, interdisciplinar e transversal que visam enriquecer a formação profissional, em uma perspectiva de integração e atualização que alinhe a vivência acadêmica à realidade profissional e social ${ }^{1}$.

Como as dificuldades observadas persistiam no colegiado do curso, foram projetadas, a partir de 2013, modificações no currículo para integração do conteúdo das disciplinas do ciclo básico em módulos, início das disciplinas do ciclo clínico a partir do segundo semestre do segundo ano com conteúdo integrado por sistemas, para que a partir de 2016 o internato pudesse ter a duração de dois anos e meio 9 . O resultado inicial não foi o esperado, visto que o ensino continuava baseado nas disciplinas e a integração não ocorreu efetivamente. Para evitar prejuízo do ensino e pelas dificuldades observadas, o núcleo docente optou, em 2014, por uma implantação mais lenta dessas modificações. Nesse mesmo ano foram publicadas as novas diretrizes curriculares para o curso de Medicina ${ }^{10}$. Estas estabeleceram que o curso deve dotar o formado em Medicina com as seguintes competências e habilidades: atenção à saúde, tomada de decisões, comunicação, administração e gerenciamento; e formar profissionais capazes de manter atualização permanente. Enfatizou-se a necessidade de atuação profissional em todos os níveis de atenção ao paciente. Com isso, as modificações implantadas em 2013 na FMABC foram ampliadas com a inclusão da atenção primária em todos os anos do curso. Foi possível também nessa reestruturação aumentar o número de atividades práticas a partir do segundo ano, com aulas para turmas menores, reivindicação antiga do corpo discente. Também se manteve o aumento do internato para dois anos e meio anos a partir de 2016.

\section{DISCUSSÃO}

Estamos em plena fase de modificações. Os excelentes resultados obtidos em avaliações externas, como Exame Nacional de Desempenho de Estudantes (Enade), prova do Conselho Regional de Medicina do Estado de São Paulo (CREMESP), exames de residência médica, nos deixam tranquilos quanto ao trabalho realizado, mas não acomodados. Como nunca, temos, hoje, condições claras para implementar uma reforma curricular que se efetive na prática com a integração e a interdisciplinaridade, pois temos um corpo docente de excelência e que tem participado e colaborado com todas as modificações propostas.

As reformas curriculares, implantadas na FMABC ao longo dos 45 anos visando à formação geral, humanística, reflexiva e ética dos graduados, têm se mostrado adequadas. As novas diretrizes curriculares nos obrigam a uma permanente reflexão e reestruturação do curso. Que venham os próximos 46 anos! 


\section{REFERÊNCIAS}

1. Faculdade de Medicina do ABC (FMABC). Projeto Pedagógico do Curso de Medicina. Santo André: FMABC; 2008.

2. Martins CB. Reforma Universitária de 1868 e a abertura para o ensino superior privado no Brasil. Educ Soc. 2009;30(106):15-35. http://dx.doi.org/10.1590/S0101-73302009000100002

3. Fernandes F. Universidade brasileira: reforma ou revolução? São Paulo: Alfa-Ômega; 1975

4. Pagliosa FL, ROS MA. O relatório Flexner: para o bem e para o mal. Rev Bras Educ Méd. 2008;32(4):492-9. http://dx.doi.org/10.1590/S0100-55022008000400012

5. Beltrame RL. A formação do médico: um debate à luz das Diretrizes Curriculares Nacionais. Tese (Doutorado) - Pontifícia Universidade Católica de São Paulo. São Paulo: 2006.

6. Tamosauskas MRG. De médico especialista a Professor de Medicina: a construção dos saberes docentes. Dissertação
(Mestrado) - Universidade Metodista de São Paulo. São Bernardo do Campo: 2003

7. Conselho Nacional de Educação. Câmara de Educação Superior. Resolução CNE/CES N ${ }^{\circ}$ 4, de 7 de novembro de 2001. Diário Oficial da União. Disponível em: http://portal.mec.gov.br/cne/ arquivos/pdf/CES04.pdf. Acesso em: 29 jul. 2015.

8. Amaral FTV, Troncon LEA. Participação de estudantes de medicina como avaliadores em exame estruturado de habilidades clínicas (Osce). Rev Bras Educ Med. 2007;31(1):81-9. http://dx.doi.org/10.1590/S0100-55022007000100011

9. Faculdade de Medicina do ABC (FMABC). Projeto Pedagógico do Curso de Medicina. Santo André: FMABC; 2012.

10. Ministério da Educação. Conselho Nacional de Educação. Câmara de Educação Superior. Resolução n 3, de 20 de junho de 2014. Disponível em: http://portal.mec.gov.br/index.php?option=com_ docman\&task=doc download\&gid=15874\&ltemid. Acesso em: 29 jul. 2015 